not belonging to European institutes, but here the administration of both the subscriptions and the page charges are handled by the journal's administrative personnel at the ESO office, naturally in close coordination with the services of the managing editor.

f) The personal subscriptions. From the outset it has been felt that apart from the institute subscriptions there should be the possibility for subscriptions by individual astronomers at a considerably reduced rate. This idea has been rigorously pursued and a category of personal subscribers established, who pay only DM 80.- per year plus postage and handling for the currently 8 volumes of 500 pages per year. Clearly, this price does not even meet the cost of production of these extra copies. The arrangement could be reached only, thanks to the collaborative attitude of Springer Verlag and the support from the sponsoring organizations. In the contract with the former, a limit has been fixed, so that the total number of personal subscribers shall not exceed a certain fraction of the number of institute subscriptions. Also, to avoid abuse, a personal subscription can be entered only by a person who certifies to be associated with an institute that has an institute subscription. Personal subscribers to the main journal receive the Supplements free of charge if they are willing to pay for the mailing and handling costs.

The administration of the personal subscriptions is handled for the main journal by the office at ESO and for the Supplements by the office of its editor in coordination with the office at ESO.

g) Editors' reports. Reports on the work of the editors are presented annually at the meetings of the Board of Directors at which they form the principal items of discussion, often leading to interesting deliberations on the policy of scientific publication in general and its evolution with a view to the future. These meetings are attended also by representatives of the publishing agent of the main journal. Among the editors' most recent items of reporting (at the meeting of 12 February 1975) was the summary of the distribution of the authorship of the articles over the various countries reproduced in the accompanying table. It appears that the authorship has, indeed, reached a world-wide character albeit with a clear European flavour.

\section{Countries of origin}

of the published articles

$\begin{array}{lrr}\text { France } & 13.6 & 15.0 \\ \text { Germany } & 10.5 & 12.6 \\ \text { Netherlands } & 6.6 & 5.0 \\ \text { Belgium } & 2.3 & 1.8 \\ \text { Scandinavia } & 1.7 & 4.3 \\ \text { Switzerland } & 1.4 & 1.2 \\ \text { Italy } & 4.0 & 6.5 \\ \text { Englanid } & 4.1 & 3.2 \\ \text { USA + Canada } & 12.9 & 11.9 \\ \text { South America } & 1.6 & 2.2 \\ \text { Other } & 5.1 & 7.9\end{array}$

Share of sponsoring

countries

$63 \%$

\title{
High Energy and Particle Physics Conference
}

\section{Palermo, Italy, 23-28 June 1975}

Six hundred high energy physicists met in Palermo for the International Conference on High Energy Physics, organized by the High Energy and Particle Physics Division of the European Physical Society. It was an intense and inspiring week, an important and welcome occasion for high energy physicists to try and assess the present achievements and future perspectives of this very rapidly developing field of physics.

Chaired by Antonino Zichichi, the Conference presented the novelty of the almost complete suppression of parallel sessions. This was generally regarded as a definite step in improving the quality of Conferences of this kind, turning them into an occasion for the community of high energy physicists at large to focus on the many different facets of the broad problematics which characterize high energy physics today.

Undoubtedly the Conference was dominated by the discovery of the new particles. Ting (MIT), one of the discoverers of what are now called the $\mathrm{J}-\psi$ particles, reported on the rapidly accumulating knowledge on these particles in high energy processes not involving electron-positron annihilation. It is in $\mathrm{e}^{+} \mathrm{e}^{-}$annihilation,

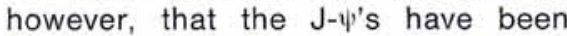

studied in most detail: Lüth (SLAC) presented the important and impressive results obtained at SPEAR, while Wolf (DESY) discussed the results so far obtained at DORIS, and finally Monacelli (Rome) reviewed the efforts made at $A D O N E$ to get a picture of the relevant parameters of the $3.1 \mathrm{GeV}$ particle, which as is well known, is just on the border of their energy range. Everybody seemed impressed about the richness and the quality of the experimental information which has been gathered on the new particles, a bare six months after their discovery.

Coming to more usual physics, Feldman (SLAC) presented the new results on the total cross section for $\mathrm{e}^{+} \mathrm{e}^{-}$ annihilation into hadrons and some interesting effects related to the strong beam polarization. A strong indication that there is some new interesting physics in lepton nucleon deep inelastic scattering was reported by Chen (U. of Michigan), who showed that the simple scaling laws discovered at SLAC seven years ago are violated at the higher FNAL energies. The message that something new is also showing up in high energy neutrino interactions was spelled out clearly in the talks by Rubbia (CERN-Harvard) and Cline (U. of Wisconsin). They re- ported evidence of new phenomena ( $\Perp$-pairs, bumps in the hadronic energy and neutrino energy loss distributions) ocurring above $30 \mathrm{GeV}$, suggesting the opening of a threshold.

On purely hadronic interactions no slackening of activity could be detected, quite the contrary. New data on total cross sections, diffractive slopes and double diffractive production have enriched our knowledge of high energy hadronic collisions, and correspondingly narrowed the possibilities for the theoretical models to account for the data. Another scaling law (Feynman's for one particle distributions) is among the casualties of this conference, a product of the vigourous ISR experimental programme at CERN, as reported by Duff (UCL). The fascinating phenomena of particle production at large transverse momenta was reviewed in a talk by Darriulat (CERN). According to the speaker one begins to unravel a certain typical jetlike structure in the final states, which suggests the presence of simple and pointlike hadronic constituents.

Some new results on the composition of final states in photo- and electro-production were the subject of the reports by Heusch (U. of $S$. Cruz) and Weber (DESY): another 
precious piece of the still very spotty mosaic of deep inelastic phenomena.

Finally the impressive results on the $(g-p)$ measurement of the moon performed at CERN (reported by Feldman) and of the $\mathrm{e}^{+} \mathrm{e}^{-}$annihilation into $\mu^{+} \mu^{-}$coming from the BCF group at Adone (reported by Palmonari (CERN)) reconfirm that Quantum Electrodynamics is alive and well, still holding up in the face of considerably accurate experiments.

The preceding is a very concise survey of the highlights of experimental physics at the Conference. How about theory? It should be said that most of the participants held the view that if these last years have been a bonanza for experimental physics, for theory they have been far less generous. Several attempts like gauge field theories unifying weak and e.m. interactions have received insufficient experimental confirmation, as reviewed by 'thooft (U. of Utrecht). However Salam (Trieste) describing the newly proposed supergauge theories, conveyed a very optimistic attitude toward the future of these appealing ideas.

The case for the deep connection between the $J-\psi$ 's and the charm quantum number, proposed long ago, was passionately made by Cabibbo (U. of Rome). However some puzzling features of the experimental findings of SPEAR and DORIS seem to present serious difficulties for the otherwise appealing "charm" scheme, as discussed by Yamagouchi (U. of Tokyo) and Mathews (Imperial College) who spoke in favour of the alternative represented by "colour" models. The vast panorama of theoretical interpretation of deep inelastic phenomena was reviewed by Preparata (CERN), who stressed the potentiality of the seemingly chaotic picture, stemming from the various theoretical models, turning itself into an accurate description of hadronic matter as seen in very small space-time regions. The status of theoretical understanding of hadronic interactions at high energy and small transverse momenta was summarized by Schwimmer (Weizmann Inst.). Finally Neven (Ecole Normale) discussed the current ideas on "quark confinement", which constitute a serious attempt on the part of today's theoretical physics to resolve the paradoxical quark behaviour.

At the enid of the Conference, after the stimulating summary made by $A$. Zichichi, people left with the distinct feeling of having spent a very rewarding week.

G. Preparata Geneva

\section{2nd International Conference on Phonon Scattering in Solids}

\section{Nottingham, England, 27-30 August 1975}

The Second International Conference on Phonon Scattering in Solids was held at Nottingham University from 27th - 30th August 1975 and was attended by 192 delegates from $15 \mathrm{Eu}$ ropean and 9 other countries. The interest in this subject has increased steadily in the last few years as new phenomena and new techniques have been discovered. One major aspect of this work is now known as phonon spectroscopy. It is directly analagous to optical or 'photon spectroscopy' (although is of course limited to frequencies below the Debye cut-off) but the selection rules are sufficiently different that many systems that cannot be seen with photons can be studied readily using phonons. The first clear evidence of resonant phonon scattering resulting from transitions between low lying energy levels was seen in 1961 using microwave phonons generated piezoelectrically - a technique now known as acoustic paramagnetic resonance - and shortly afterwards using thermal phonons in thermal conductivity experiments. It then soon became clear that phonon spectroscopy was going to be a valuable addition to techniques for investigating systems with energy level separations in the frequency range up to a few $\mathrm{THz}$, that is the far infra-red range and below of optical spectroscopy.

The thermal conductivity technique despite its very modest resolution is still in common use because of its ability to observe resonances over a very wide frequency range $\left(\sim 10^{\circ}\right.$ $10^{12} \mathrm{~Hz}$ ). In magnetic and electric fields or on application of stress, it can be used to obtain information on the movement of levels with field or lattice coupling constants and if the levels are moved until two levels cross or until two pairs of levels are equally spaced (frequency crossing) spectroscopic information can be achieved with a resolution of better than $1 \%$. The use of thermal phonons has been further extended by using heat pulses and time of flight techniques to separate different polarizations. A number of other powerful techniques have also been developed. In one of these, the phonons are generated by biasing a superconducting tunnel junction evapored on to the dielectric sample. The electrons injected across the junction lose energy by emitting phonons, and by modulating the bias voltage 'monochromatic' phonons can be selected from the emission spectrum. This technique can be very sensitive and has a resolution of $\check{<} 1 \%$. Selective quantum detectors have also been developed using magnetic energy levels, stress, dependent levels and tunnel junctions. Also the piezoelectric technique has been extended to above $1 \mathrm{THz}$ using far infra-red lasers. These techniques have been used in spectroscopic studies of magnetic ions, localised electrons and holes in semiconductors, two level systems in amorphous materials, molecular defects and so on. But their application is very much wider than spectroscopy and includes investigations of phonon transmission across interfaces (the Kapitza problem), phonon echoes, cooperative and critical effects, liquid helium, superconductors, anharmonic effects and many others.

The Nottingham conference followed conferences in Paris and St Maxime in 1972 organized by Albany and by Zylberstejn respectively. It was clear that the subject had advanced considerably in these three years. The Kapitza conductance problem was one area discussed with great enthusiasm. It now seems very likely that the transmission of phonons across interfaces between any two materials can be accounted for by classical acoustic theory at frequencies below $\sim 1 \mathrm{GHz}$. But at higher frequencies, $\sim 10 \mathrm{GHz}$ and above, although the theory still works well for most interfaces, for some, in which one of the materials is gaseous, liquid or solid helium or solid hydrogen, it very badly underestimates the transmission observed. In one paper measurements using monochromatic phonons, and so providing much better resolution than earlier work using thermal phonons, showed the presence of a rapid decrease in phonon reflectivity between 20 and $50 \mathrm{GHz}$ as the frequency was increased. In another paper it was shown that some phonons ( $10 \%$ at $1 \mathrm{~K}$ ) 